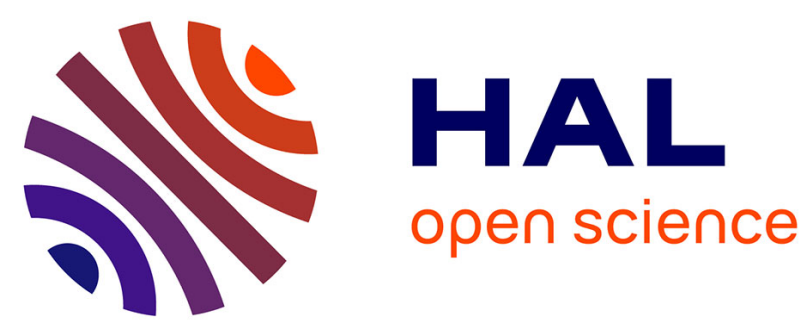

\title{
Solvation Free Energies and Adsorption Energies at the Metal/Water Interface from Hybrid Quantum-Mechanical/Molecular Mechanics Simulations
}

\author{
Paul Clabaut, Benjamin Schweitzer, Andreas W. Götz, Carine Michel,
} Stephan N. Steinmann

\section{To cite this version:}

Paul Clabaut, Benjamin Schweitzer, Andreas W. Götz, Carine Michel, Stephan N. Steinmann. Solvation Free Energies and Adsorption Energies at the Metal/Water Interface from Hybrid QuantumMechanical/Molecular Mechanics Simulations. Journal of Chemical Theory and Computation, 2020, 16 (10), pp.6539-6549. 10.1021/acs.jctc.0c00632 . hal-02998070

\section{HAL Id: hal-02998070 https://hal.science/hal-02998070}

Submitted on 10 Nov 2020

HAL is a multi-disciplinary open access archive for the deposit and dissemination of scientific research documents, whether they are published or not. The documents may come from teaching and research institutions in France or abroad, or from public or private research centers.
L'archive ouverte pluridisciplinaire HAL, est destinée au dépôt et à la diffusion de documents scientifiques de niveau recherche, publiés ou non, émanant des établissements d'enseignement et de recherche français ou étrangers, des laboratoires publics ou privés. 


\title{
Solvation Free Energies and Adsorption
}

\section{Energies at the Metal/Water Interface from Hybrid QM-MM Simulations}

Paul Clabaut, ${ }^{\dagger}$ Benjamin Schweitzer, ${ }^{\dagger}$ Andreas W. Götz, ${ }^{\ddagger}$ Carine Michel, ${ }^{+}$and Stephan N. Steinmann*,

+Univ Lyon, Ecole Normale Supérieure de Lyon, CNRS Université Lyon 1, Laboratoire de Chimie UMR 5182, 46 allée d'Italie, F-69364, LYON, France

$\ddagger$ San Diego Supercomputer Center, University of California San Diego, La Jolla, California 92093, USA

E-mail: stephan.steinmann@ens-lyon.fr

Phone: (+33)4 72728155

\begin{abstract}
Modeling adsorption at the metal/water interfaces is a corner-stone towards an improved understanding in a variety of fields from heterogeneous catalysis to corrosion. We propose and validate a hybrid scheme that combines the adsorption free energies obtained in gas phase at the DFT level with the variation in solvation from the bulk phase to the interface evaluated using a molecular mechanics based alchemical transformation, denoted MMsolv. Using the GAL17 force field for the platinum/water interaction, we retrieve a qualitatively correct interaction energy of the water solvent at the interface. This interaction is of near chemisorption character and thus challenging, both for the alchemical transformation, but also for the fixed point-charge electrostatics.
\end{abstract}


Our scheme passes through a state characterized by a well-behaved physisorption potential for the $\mathrm{Pt}(111) / \mathrm{H}_{2} \mathrm{O}$ interaction to converge the free energy difference. The workflow is implemented in the freely available SolvHybrid package. We first assess the adsorption of a water molecule at the $\mathrm{Pt} /$ water interface, which turns out to be a stringent test. The intrinsic error of our QM-MM hybrid scheme is limited to 6 $\mathrm{kcal} \cdot \mathrm{mol}^{-1}$ through the introduction of a correction term to attenuate the electrostatic interaction between near-chemisorbed water molecules and the underlying Pt atoms. Next, we show that the MMsolv solvation free energy of $\mathrm{Pt}\left(-0.46 \mathrm{~J} \cdot \mathrm{m}^{-2}\right)$ is in good agreement with the experimental estimate $\left(-0.32 \mathrm{~J} \cdot \mathrm{m}^{-2}\right)$. Furthermore, we show that the entropy contribution at room temperature is roughly of equal magnitude as the free energy, but with opposite sign. Finally, we compute the adsorption energy of benzene and phenol at the $\mathrm{Pt}(111)$ / water interface, one of the rare systems for which experimental data are available. In qualitative agreement with experiment, but in stark contrast with a standard implicit solvent model, the adsorption of these aromatic molecules is strongly reduced (i.e., less exothermic by $\sim 30$ and $40 \mathrm{kcal} \cdot \mathrm{mol}^{-1}$ for our QM/MM hybrid scheme and experiment, respectively, but $\sim 0$ with the implicit solvent) at the solid/liquid compared to the solid/gas interface. This reduction is mainly

due to the competition between the organic adsorbate and the solvent for adsorption on the metallic surface. The semi-quantitative agreement with experimental estimates for the adsorption energy of aromatic molecules thus validates the soundness of our hybrid QM-MM scheme.

\section{Introduction}

Assessing the impact of water at the metal/liquid interface is a challenging task but mandatory to understand and predict numerous interfacial processes from heterogeneous catalysis to water treatment, metal plating and corrosion. The lack of extensive experimental data is clearly detrimental to validate proposed methods. As it serves as both, a theoretical and experimental model system for the understanding of metal/water in- 
terfaces, the platinum/water interface is the only exception in this respect. Pt is well characterized in surface science studies ${ }^{1,2}$ and highly relevant to heterogeneous catalysis and electrocatalysis. ${ }^{3-7}$ Furthermore, $\mathrm{Pt}(111) /$ water is one of the few solid/liquid interfaces for which a couple of experimental solvation energies are available. ${ }^{8-10}$ We herein will rely on this exceptionally well-characterized interface to validate our general approach by comparing our computed adsorption energies at the solid/liquid interface to the available experimental values.

Over the last twenty years, several approaches have been developed to computationally account for solvation effects at the metal/water interface. In the microsolvation scheme, a limited number of water molecules are co-adsorbed with the adsorbate of interest. ${ }^{11-15} \mathrm{To}$ achieve a balanced description of solvation along a reaction profile, these water molecules have to be carefully chosen and placed. In addition to being cumbersome, microsolvation only allows to retrieve the short range part of the solvation effects, also called direct solvation effects. The indirect solvation effects, i.e., the modified electrostatic interactions as well as the entropy of solvation are completely neglected in this approach. ${ }^{16-20}$ The implicit solvation models such as the polarizable contiuum model, $\mathrm{PCM},{ }^{21}$ are precisely designed to retrieve the indirect solvation effects and are most successful to recover the average electrostatic interaction between solutes and the solvent which is described by its dielectric constant. However the lack of a database of reference solvation energies at the water/metal interface severely limits the parametrization and validation of implicit solvation models for the metal/liquid interface. The cluster-continuum model, where the direct (local) solvation effect is described by microsolvation and the indirect (bulk) solvation is approximated by the dielectric constant try to combine the best of both worlds. ${ }^{15}$ Alternatively the full water phase can be computed. However, phase-space sampling with an accurate energy description is the main issue with these approaches. In an early study along these lines a short molecular dynamics (MD) simulation was performed prior to the optimization of snapshots, yielding a metal/amorphous ice-like interface. ${ }^{22}$ Similar studies 
for various metal surface/adsorbate combinations have been performed with widely varying number of water molecules and sampling length. ${ }^{23,24}$ However, these studies have not assessed to which extent they actually mimic a metal/liquid water interface. Hybrid quantum-mechanical/molecular mechanics (QM/MM) approaches have also been suggested but have so far not been compared to the rare experimental data. ${ }^{25,26}$ Our twostep hybrid QM-MM level, that we had called MM-FEP, ${ }^{27}$ consists in describing the surfaceadsorbate interaction via a DFT optimization and accounting for the solvation effects of the frozen DFT geometry through free energy perturbation (FEP) exploiting efficient molecular mechanics. This allows to retrieve all the major solvation effect, including the competition between water and the adsorbate for adsorption on the surface. Independently and in parallel to our work, Getman and co-workers developed a similar approach to asses adsorption free energies of $\mathrm{CO}$ and sugars on $\mathrm{Pt}$ (111) and compared it with implicit solvation and ice-like structures optimisations. ${ }^{28,29}$ The accuracy of any kind of $\mathrm{QM} / \mathrm{MM}$ heavily relies on the quality of the forcefield used to sample the phase-space of the solid/liquid interface. Even though several forcefields enjoy great popularity, ${ }^{30-33}$ it is only recently that we have introduced GAL17, the first qualitatively correct force field for the interaction between a water molecule and a $\mathrm{Pt}(111)$ surface. ${ }^{34}$ This force field has subsequently been generalized to other metal surfaces. ${ }^{35}$ Thus, we are now ready to tackle the solvation free energies at the $\operatorname{Pt}(111)$ surface with a reliable force field.

Alchemical transformations such as FEP or thermodynamic integration (TI) rely on comparably small changes in the Hamiltonian, most suitable for non-covalent interactions. However, the GAL17 force field correctly describes the interaction between water molecules and the metal surface by a "strong" interaction. Therefore, special attention needs to be paid during the alchemical transformation in order to avoid dependence of the results on the starting point (hysteresis), which is the first challenge addressed herein. The second challenge is how to make the chemisorption-like potential of GAL17 compatible with point-charge electrostatics. When developing GAL17, electrostatics between the 
surface and the water molecules have not been explicitly taken into account, i.e., they are part of the interaction potential but not expressed in terms of point charges. This is comparable with biological force fields, where point-charges are not part of bond or angle terms. However, when adsorbates are present on the surface, the point charges are crucial for retrieving solvation energies at the metal/liquid interface. Nevertheless, double-counting needs to be avoided between GAL17 and the point-charges.

In this work, we perform computations in the spirit of MM-FEP, but adapting it according to the two identified challenges when using GAL17 in alchemical transformations. The thus obtained solvation energies are called "MMSolv" for molecular mechanics solvation energies. When combined with DFT interaction energies to give adsorption energies at the metal/liquid interface, we call the level of theory "hybrid", to indicate the use of a QM and a MM constituent. We implement the approach in an easy-to-use package, SolvHybrid. By the comparison with experimental adsorption energies of benzene and phenol at the $\mathrm{Pt}(111)$ /water interface, we demonstrates a semi-quantitative accuracy of the devised hybrid level of theory. Given suitable force fields, SolvHybrid is applicable to the determination of solvation energies at any solid/liquid interface. Therefore, we expect SolvHybrid to become a valuable tool for mechanistic investigations in heterogeneous (electro-)catalysis and for other processes at the solid/liquid interface.

\section{Theory}

\subsection{SolvHybrid: Principles and Workflow}

The SolvHybrid package aims at computing the adsorption free energy of a molecule $M$ on a surface $S$ in a solvent using a hybrid scheme. This quantity, named here $\Delta_{a} G^{\text {solv }}$, can be decomposed in two terms:

$$
\Delta_{a} G^{\text {solv }}=\Delta_{a} G^{v a c}+\Delta_{a} \Delta_{h} G
$$


where $\Delta_{a}$ designates the free energy difference of adsorption of $M$ on $S$ and the superscript solv and vac refer to the process in solvent (here water) and vacuum or gas-phase, respectively and $\Delta_{h}$ collects all solvent effects ( $h$ stands here for hydration). $\Delta_{a} \Delta_{h} G$ formally also includes the change of adsorbate-surface interaction due to the co-adsorption of solvent molecules and the polarization of the adsorbate due to the presence of the solvent.

The first contribution $\left(\Delta_{a} G^{v a c}\right)$ is the Gibbs Free Energy of adsorption of the molecule $M$ on the surface $S$ computed in the gas-phase. To a good approximation it does not require extensive phase-space sampling ${ }^{36}$ and can, therefore, be evaluated by standard DFT optimizations, here performed using VASP. ${ }^{37,38}$ SolvHybrid computes the hybrid adsorption energy, combining the DFT energy $\Delta_{a} G^{v a c}$ with the MMSolv energy correction term $\Delta_{h} \Delta_{a} G$. Hence, $\Delta_{a} G^{v a c}$ and the corresponding optimized geometries, together with their Hirshfeld charges, of $M$, the surface $S$ and the adsorbate-surface assembly (M@S) constitute the basis of SolvHybrid computations.

The MMSolv term $\left(\Delta_{a} \Delta_{h} G\right)$ is the variation of the free energy of solvation $\left(\Delta_{h} G\right)$, during the adsorption process, i.e., from bulk solution to the interface. The solvation effects derive mostly from the reorganization of the solvent around the adsorbate (often referred to as the cavitation energy), the replacement of water molecules adsorbed on the surface by the adsorbate and from the change in interactions between the molecule in bulk solution and at the interface, i.e., the change in polarization. Within the SolvHybrid package, the computation of $\Delta_{a} \Delta_{h} G$ is performed with molecular mechanics (MM) within the framework of thermodynamic integration (TI) as implemented in AMBER. ${ }^{39,40}$ The principles of the alchemical TI are summarized in section S1 of the SI. In principle, the MM requires the evaluation of three different types of interactions: (i) metal/adsorbate, (ii) water/adsorbate, (iii) water/metal. Since no accurate and general force field exists for the interaction between adsorbates and metal surfaces, we set this interaction to zero and keep the DFT determined geometry frozen during the MM computations. As a compensation, $\Delta_{a} G^{v a c}$ is, of course, added in the end to obtain $\Delta_{a} G^{\text {solv }}$ (see Eq. 1). The 
most stable adsorption configuration at the solid/liquid interface can, nevertheless, be determined if the solvation energy is computed for different (gas-phase) configurations, i.e., the configurational sampling is factored out of the TI and only performed in vacuum or implicit solvent. The water/metal interaction is conveniently described by the GAL17 force-field. ${ }^{34}$ Finally, the water/adsorbate interaction is approximated by established Lennard-Jones parameters ${ }^{41}$ and atomic charges computed with DFT. ${ }^{42}$ This approach approximates the polarization of the adsorbate by the presence of the solvent according to the scheme devised by Jorgensen and co-workers, ${ }^{43}$ but neglects the modification of adsorbate-surface interaction due to the co-adsorption of solvent molecules.

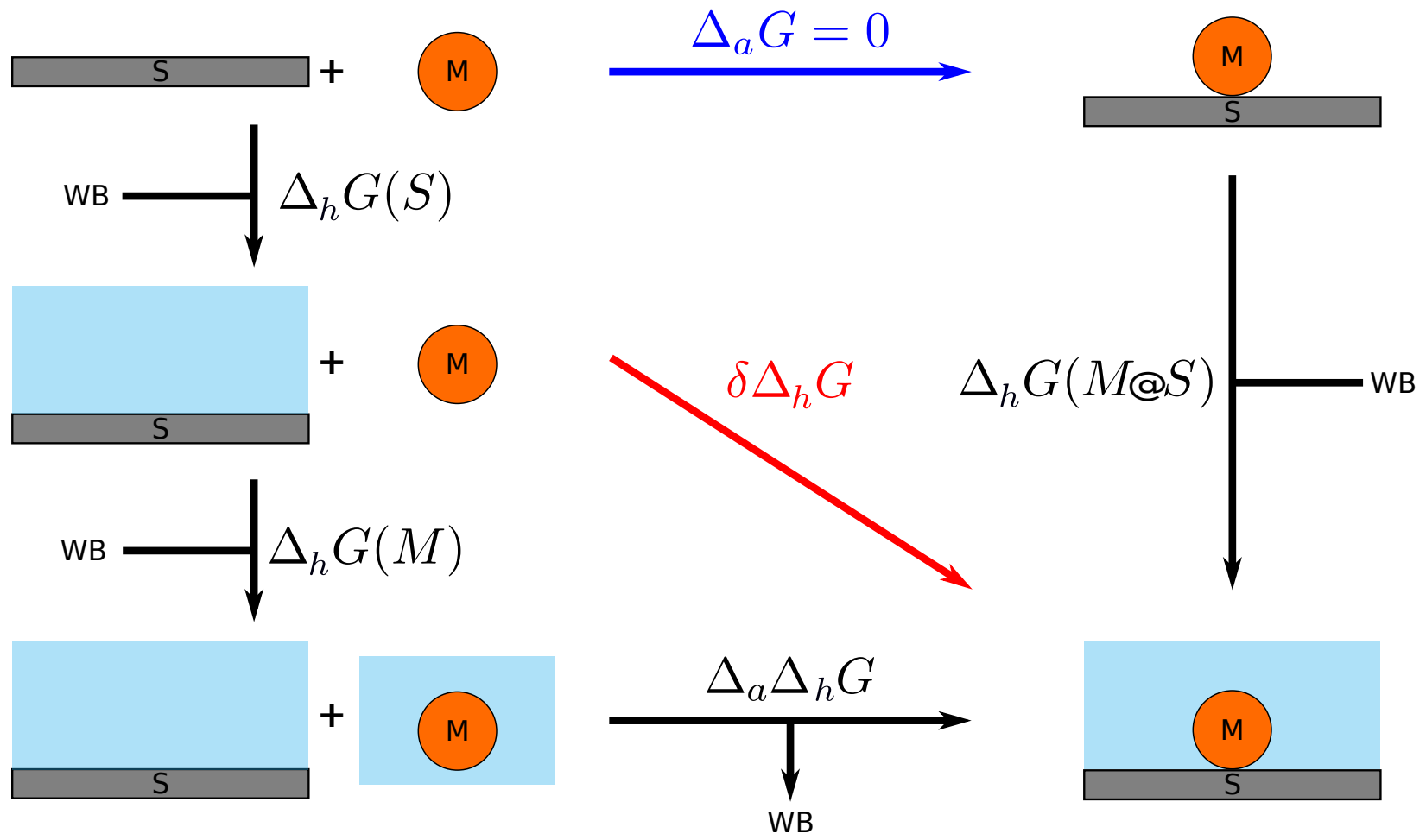

Figure 1: Thermodynamic cycle used to determine $\Delta_{a} \Delta_{h} G$. $M$ represents the studied molecule, $S$ the metallic surface, $M @ S$ the molecule adsorbed on the surface, and WB a water box of a sufficient size. $\Delta_{h} G$ expresses the solvation free energy of a compound, while $\Delta_{a} G$ is the adsorption free energy associated to $M+S \rightarrow M @ S$. $\delta \Delta_{h} G$ is defined as the free energy change associated with the transformation depicted by the red arrow.

Following our previous work, ${ }^{27}$ we thus exploit the thermodynamic cycle of Fig. 1 to evaluate $\Delta_{a} \Delta_{h} G$. In principle, $\Delta_{a} \Delta_{h} G$ can be computed in three ways: (i) by direct transfor- 
mation (horizontal black line), (ii), following the rectangle of black arrows, exploiting the "standard" thermodynamic cycle, i.e., $\Delta_{a} \Delta_{h} G=\Delta_{a} G+\Delta_{h} G(M @ S)-\Delta_{h} G(S)-\Delta_{h} G(M)$, with $\Delta_{a} G$ being equal to zero in the chosen computation framework (MM with no interaction between the molecule and the surface), and (iii) invoking the triangle with the red, diagonal arrow, i.e., $\Delta_{a} \Delta_{h} G=\delta \Delta_{h} G-\Delta_{h} G(M)$. The direct transformation (i) is difficult to achieve as it requires the simultaneous presence of two systems in the initial state, i.e., a very large water box to mimic separated systems. The terms $\Delta_{h} G(S)$ and $\Delta_{h} G(M @ S)$, required for process (ii), present an abrupt chemical change (the appearance of a full metallic surface in the bulk water), that is computationally costly to converge. As we have shown previously, ${ }^{27}$ cycle (iii) avoids these issues and introduces a smoother transition, $\delta \Delta_{h} G$, between the empty surface $S$ and $M @ S$, both immersed in water.

The two transformations corresponding to $\delta \Delta_{h} G$ and $\Delta_{h} G(M)$ are, therefore, driven by the SolvHybrid package, in order to determine $\Delta_{a} \Delta_{h} G$. Note, that the surface part of $M @ S$ and $S$ is slightly different. Indeed, the deformation, polarisation and electron transfer induced by the adsorption of the molecule $M$ is computed at DFT level, taken in account within $\Delta_{a} G^{v a c}$ and is responsible for the different geometries and charges of the $S$ (sub-)system.

In summary, the complete adsorption energy of a compound at the solid/liquid interface is computed by SolvHybrid as:

$$
\Delta_{a} G^{\text {solv }}=\Delta_{a} G^{v a c}+\Delta_{a} \Delta_{h} G=\Delta_{a} E_{D F T}^{v a c}+\delta \Delta_{h} G_{M M}-\Delta_{h} G_{M M}(M)
$$

\subsection{Electrostatic interactions between the solvent and the metallic sur- face}

The standard implementation of Coulombic interactions in AMBER for periodic systems relies on the use of the Particle-Mesh-Ewald (PME) method. While PME is highly efficient, it is intrinsically more complex than the direct space evaluation of the Coulomb interactions. 
An alternative to PME is to use "shifted" Coulombic interactions $\left(E_{C}^{\text {shift }}\right)$ in direct space, which make the electrostatic interaction compatible with a cut-off distance $r_{c u t}$. The role of the shift-function is to render the evaluation of the Coulomb interactions in direct space compatible with periodic boundary conditions while avoiding discontinuities in the energy or forces. Here, we rely on the following "shifted" Coulombic interactions, initially introduced in the CHARMM community: ${ }^{44-46}$

$$
E_{C}^{\text {shift }}\left(r_{i j}, q_{i}, q_{j}\right)=q_{i} q_{j} \cdot \frac{1}{r_{i j}} \cdot\left(1-\frac{r_{i j}^{2}}{r_{c u t}^{2}}\right)^{2}
$$

where the squared term in parenthesis corresponds to the shift function, $r_{i j}$ is the internuclear distance between atoms $i$ and $j . q_{i}$ and $q_{j}$ are the respective atomic charges in suitable units. Beyond the cutoff distance $r_{c u t}$, the Coulomb interactions are no longer evaluated. We use this expression for all charges that do not involve metal atoms.

The reason why we herein prefer the simplicity of Eq. 3 compared to PME is the following: In contrast to standard non-bonded parameters, the GAL17 force field captures weak chemisorption of water molecules with the metal surface. Therefore, the internuclear distance between $\mathrm{Pt}$ and $\mathrm{O}$ is rather short (about $2.5 \AA$ ) in the minimum. Furthermore, since GAL17 is a non-polarizable force field (the polarization energy that is easily recoverable being only about $1 \mathrm{kcal} \cdot \mathrm{mol}^{-1}$ for a single water molecule ${ }^{47}$ ), we had assumed a zero atomic charge on Pt during its development. However, when adsorbates are present on the surface, we necessarily also need to take atomic charges into account to describe the electrostatic interaction between the solvent and the adsorbate. Since we determine these charges from first principles for the $M @ S$ system, they unavoidably lead to partial charges on the Pt atoms. To make GAL17 compatible with atomic charges on $\mathrm{Pt}$, we tested and adopted a strategy inspired by standard force fields, where the Coulomb interaction is set to zero for 1-2, 1-3 and scaled down for 1-4 covalently bonded neighbors. ${ }^{44}$ In standard (fixed topology) force fields, these short-ranged Coulomb interactions are excluded (or 
scaled down) since the corresponding interaction terms are already implicitly taken into account through the chemical bonds. Since quantum-mechanical effects are dominating at such short internuclear distances, the variation in the Coulomb energy of point charges would, anyway, not be physically relevant. However, GAL17 is a "semi-reactive" force field in the sense that the water/Pt topology is not fixed, even though the internal connectivity of the water molecules and the metal surface cannot change, preventing water dissociation and metal dissolution. Due to the flexible water/Pt topology, we cannot simply exclude the corresponding $\mathrm{Pt}-\mathrm{O}$ and $\mathrm{Pt}-\mathrm{H}$ pairs, but smoothly switch off the Coulombic interactions at short distances, which is nearly trivial in direct space. This is achieved by introducing a Fermi damping function for the $\mathrm{Pt}-\mathrm{H}_{2} \mathrm{O}$ Coulombic interactions, resulting in:

$$
E_{C}^{s h i f t}\left(r_{P t, j}, q_{P t}, q_{j}\right)=q_{P t} q_{j} \cdot \frac{1}{r_{P t, j}} \cdot\left(1-\frac{r_{P t, j}^{2}}{r_{c u t}^{2}}\right)^{2} \cdot\left(\frac{1}{1+\exp \left(-\sigma\left(\frac{r_{P t, j}}{r_{0}}-1\right)\right)}\right)
$$

where $\sigma$ and $r_{0}$ are the adjustable parameters of the Fermi-damping, corresponding respectively to the switching slope and the characteristic distance of the short-range correction. The two damping parameters were adjusted to minimize the highest $\Delta_{a} \Delta_{h} G$ of water molecule on the $\mathrm{Pt}$ (111) surface, determined as presented in section 4.1. The final parameters were 25 and $3.3 \AA$, for $\sigma$ and $r_{0}$, respectively. The resulting Coulomb interaction is shown in Fig. 2, where it is compared to the bare and shifted Coulomb operator.

\section{Computational Details}

\subsection{Molecular mechanics simulations}

The MUSIC module for AMBER, ${ }^{39}$ implementing the GAL17 forcefield, was used to describe water-Pt interactions along with the set of published parameters. ${ }^{34} \mathrm{~A}$ second version of the forcefield, dubbed "sGAL17" for soft GAL17, was also used. It consists in 


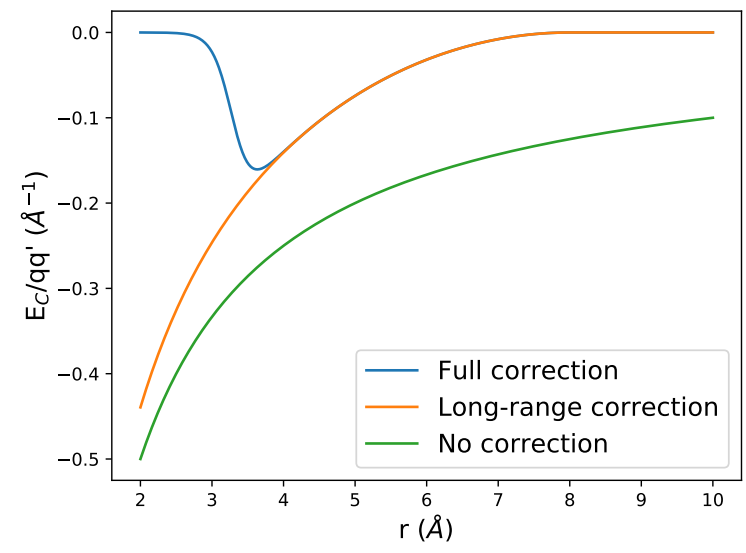

Figure 2: Distance dependence of the electrostatic interactions, renormalized by the partial charges $q_{i}, q_{j}$. The green line corresponds to the bare Coulomb operator $\left(\frac{1}{r}\right)$, while the orange curve is the shifted electrostatic interaction according to Eq. 3. The blue line results from the short-range damping of Eq. 4. $r_{c u t}, \sigma$ and $r_{0}$ were set to $8.0 \AA$; 25 and $3.3 \AA$, as used for all our computations.

exactly the same functional form, but all parameters with energy as a unit are divided by four.

Water-water interactions were computed via the TIP3P water model. ${ }^{48}$ All LennardJones interactions other than TIP3P are taken from the UFF force field. ${ }^{41}$ In all computations, the non-aqueous part of the system $(S, M @ S$ and $M)$, was frozen using the belly algorithm of AMBER. The shake algorithm was exploited to constrain the geometry of the water molecules, allowing to use a time step to $2 \mathrm{fs} .{ }^{49} .50$ The pressure was anisotropically scaled, i.e., only the out-of-plane unit-cell vector was allowed to change by adapting the default Berendsen barostat of AMBER ${ }^{51}$ accoordingly.

The thermodynamic integration was carried out using the default settings of AMBER17, including the softcore potential ${ }^{52,53}$ when performing the actual alchemical change.

\subsection{DFT computations}

All geometries for the molecule $(S)$, the molecule adsorbed on the metallic surface $(M @ S)$ and the bare metallic surface $(M)$ were optimized with VASP ${ }^{37}, 38$ using the conjugate- 
gradient algorithm. The PBE generalized gradient approximation ${ }^{545}$ was chosen as the exchange-correlation functional, complemented by the $\mathrm{dDsC}$ dispersion correction, ${ }^{56}$ a setup that has previously been validated against experimental gas-phase adsorption energies. ${ }^{57}$ The plane-wave energy cutoff was set to $400 \mathrm{eV}$. The electron-ion interactions were described by the PAW formalism. ${ }^{58,59}$ A $\Gamma$-centered $3 \times 3 \times 1$ Monkhorst-Pack Kpoint ${ }^{60}$ grid was used to sample the Brillouin zone. All slabs were built as initially ideal $\mathrm{p}(4 \times 4) \operatorname{Pt}(111)$ cells with 4 layers. Only the two topmost layers were allowed to relax during the optimizations. All geometries are available in the supporting information. The VASPsol module ${ }^{21,61}$ was used to assess the effect of an implicit solvent for comparison. The default settings for cavitation energy are used, and the plane-wave energy cutoff was increased to $600 \mathrm{eV}$. When a Pt slab was present, a dipole correction was applied in the out-of-plane direction, ${ }^{62}$ to avoid solvating a macroscopic dipole. ${ }^{18}$

\subsection{MM setup via SolvHybrid}

SolvHybrid allows to setup the MM computations automatically. In practice, it builds $3 \times 3$ supercells (other multipliers are supported) with respect to the DFT unit cells to extend the lateral size of the surface well beyond the MM cut-off distance and to improve statistics. Atomic charges for the surface and molecules are extracted from Hirshfeld charges of the VASP computations, ${ }^{42}$ re-scaled as CM5 charges $^{63}$ and multiplied by the recommended factor of 1.27. ${ }^{43}$ Single-point gas-phase MM energies ( $E_{M M}^{v a c}$ ) are computed for each system (S, $M$, and $M @ S)$. The $M @ S$ and $M$ systems are then solvated by a $\sim 30 \AA$ thick TIP3P water box, ${ }^{48}$ via the algorithm of leap from the AmberTools. The resulting orthorhombic box completely surrounds the system. Water molecules not above the surface are then removed so that the final water box matches the original in-plane dimensions of the $3 \times 3$ supercells. The water boxes of $M @ S$ and $M$ are then minimized for 2000 cycles to diminish highly repulsive forces. An exact copy of the water box of the $M @ S$ system is then extracted to be

place on top of $S$. This leads to two systems ( $\mathrm{S}$ and $M @ S$ ) that only differ by the coordinates 
of the atoms that are being transformed during the TI, as requested by AMBER.

\subsection{Settings for thermodynamic integrations}

As described in section 2.1, SolvHybrid performs two independent TIs. $\mathrm{TI}_{1}$ connects $M @ S$ to $\mathrm{S}:(\mathrm{M} @ S)^{\text {solv }} \rightarrow(\mathrm{S})^{\text {solv }}$ required to evaluate $\delta \Delta_{h} G$. $\mathrm{TI}_{2}$ connects $\mathrm{M}$ to bulk liquid water: $(\mathrm{M})^{\text {solv }} \rightarrow(\varnothing)^{\text {solv }}$ and is the main constituent for $\Delta_{h} G(M)$, the MMsolv solvation energy of the entity $M$.

When performing the TIs, the self-energy of the fragments that are alchemically transformed are also annihilated/created. Hence, to comply with the thermodynamic cycle of Fig. 1, the corresponding energies in vacuum have to be subtracted, so that the effective MM adsorption energy in vacuum $\Delta_{a} E_{M M}^{v a c}$ does not contribute to the final result:

$$
\begin{aligned}
\Delta_{a} \Delta_{h} G & =\delta \Delta_{h} G_{M M}-\Delta_{h} G_{M M}(M) \\
& =\left(-\Delta_{T I_{1}} G-E_{M M}^{v a c}(M @ S)+E_{M M}^{v a c}(S)\right)-\left(-\Delta_{T I_{2}} G-E_{M M}^{v a c}(M)\right) \\
& =\Delta_{T I_{2}} G-\Delta_{T I_{1}} G-\Delta_{a} E_{M M}^{v a c}
\end{aligned}
$$

Finally, we can write the hybrid adsorption energy in solution as:

$$
\Delta_{a} G^{s o l v}=\Delta_{a} E_{D F T}^{v a c}-\Delta_{a} E_{M M}^{v a c}+\Delta_{T I_{2}} G-\Delta_{T I_{1}} G
$$

Following standard practice in AMBER, each TI is separated in several partial transformations, which we call "step" herein. Each step is itself divided into 11 windows, corresponding to different values of the mixing parameter $\lambda$. For each window, individual minimization, heating and equilibration to $300 \mathrm{~K}$ and production at 1 bar are performed.

The minimization is carried out for 100 cycles at constant volume. Heating from 5 to $300 \mathrm{~K}$ is achieved over $200 \mathrm{ps}$, i.e., at a heat rate of $1.475 \mathrm{~K} \cdot \mathrm{ps}^{-1}$, with a thermostat 
time constant of 1 ps while the pressure is kept constant at 1 bar. Equilibration is carried out for $100 \mathrm{ps}$ in the NPT ensemble, keeping the the temperature at $300 \mathrm{~K}$ by a Langevin thermostat with a collision frequency of $2 \mathrm{ps}^{-1} \cdot{ }^{64} 200 \mathrm{ps}$ of dynamics under the same conditions are used as the production phase.

The steps constituting the TIs are: (i) Discharge of the non aqueous part of $M @ S$, i.e., scaling the atomic charges of $M @ S$ progressively to zero, (ii) For $M @ S$ with no atomic charges, transforming the GAL17 forcefield to sGAL17, (iii) Alchemical change from M@S to $S$ with no atomic charges on $S$ and $M @ S$ and with sGAL17 for the water/Pt interaction, (iv) For $S$, transform the sGAL17 to the GAL17 forcefield, (v) Recharge of the non-aqueous part of $S$, i.e., scaling atomic charges of $S$ from zero to their physical values. We find that step (ii) and step (iv) are necessary to ensure reproducible results, avoiding hysteresis with windows being trapped in meta-stable states for several nanoseconds.

The discharge (i), recharge (v), and alchemical (iii) steps are conducted on 9 evenly spaced windows with $\lambda$ ranging from 0.1 to 0.9 , plus 2 windows at $\lambda$ equal to 0.005 and 0.995. The softening of GAL17 (ii) and its reverse (iv) are performed in 11 logarithmicspaced (spacing following $\ln (x)$ ) and decreasing logarithmic-spaced windows (spacing following $1-\ln (x))$ ranging from 0.005 to 0.995 , which reflects the exponential shape of $\left\langle\frac{\partial V(\lambda)}{\partial \lambda}\right\rangle_{\lambda}$ during these transformations (see Fig. S1 and Fig. S2 in the supporting information).

Using an algorithm from Steinbrecher, ${ }^{65}$ free energy variations are gathered over the last 200 ps of each window, numerically integrated for each step and summed to the corresponding total free energy change.

\section{Results and discussion}

Having described the developed hybrid method in section 2.1, we now turn to the actual results obtained when combining DFT in vacuum with MMsolv solvation energies. By 
computing the solvation energy of $\mathrm{H}_{2} \mathrm{O} @ \mathrm{Pt}(111)$, we first investigate the intrinsic error, which is committed by the uneven description for adsorbates and the solvent. Then, we move to the solvation energy of the $\mathrm{Pt}(111)$ surface in order to obtain rough estimates of the energetic costs to displace water molecules from the surface. Finally, we validate our method by assessing the adsorption free energy of benzene and phenol at the $\mathrm{Pt}(111)$ /water interface, for which experimental estimates are available.

\section{1 $\quad \mathrm{H}_{2} \mathrm{O} @ \mathrm{Pt}(111)$ solvation energies to monitor inconsistencies}

To estimate the inconsistencies of the scheme due to the dual DFT/MM description, we here evaluate the adsorption free energy of water in the water solvent. The considered transformation is an exchange between two water molecules, one which is in the bulk solvent, and the other one is adsorbed on the surface. Hence, the energy balance of this exchange should be zero. Practically, in the MMSolv scheme, the adsorption of a water molecule is described by DFT, i.e., via a DFT geometry optimization and $\Delta_{a} E_{D F T}^{v a c}$, just like for any other adsorbate. Simultaneously, the desorption of MM-described water molecule(s) makes space for the DFT adsorbate. We consider this exchange as the worstcase scenario for the present scheme since it implies an exchange between two molecules that are supposedly identical, but are described by two different levels of theory. Furthermore, the adsorbate is small and interacts strongly with the solvent, so that there is barely room for error cancellation. The resulting adsorption free energy, compared to the expected zero energy balance, will, therefore, be particularly sensitive to discrepancies between the two levels of theory. This adsorption free energy was also assessed as a function of the distance of the water molecule from the surface. This evaluates the committed error, the smoothness and convergence behavior toward the expected zero energy result far away from the surface.

We will here use $\mathrm{H}_{2} \mathrm{O}^{\text {DFT }}$ to distinguish it from the MM water molecules. In this case, $\Delta_{a} G^{\text {solv }}$ represents the free energy required to replace a MM water molecule on the surface 
by $\mathrm{H}_{2} \mathrm{O}^{\text {DFT } @ P t(111) ~ a n d, ~ s i m u l t a n e o u s l y ~ m a k e ~} \mathrm{H}_{2} \mathrm{O}^{\text {DFT }}$ disappear from the bulk water. $\mathrm{H}_{2} \mathrm{O}^{\text {DFT }}$ is distinct from the MM water molecules in three ways: (i) its interaction with the surface is treated at the DFT level and consists in $\Delta_{a} E_{D F T}^{v a c}$, (ii) it polarizes the platinum surface during its adsorption while the MM water molecules do not, (iii) it is frozen during the TI and therefore can neither be exchanged with other water molecules, nor move to optimize the organisation of the interfacial structure of water.

$\Delta_{a} G^{\text {solv }}\left(\mathrm{H}_{2} \mathrm{O}\right)$ is used to determine the effectiveness of the Coulomb correction described in section 2.2: Fig. 3 compares the results obtained with the standard (shifted) Coulomb interactions in orange (Eq. 3) with the short-range corrected interactions (in blue) of Eq. 4. Note, that the results for Particle Mesh Ewald are of equivalent quality to the orange lines of the standard shifted Coulomb scheme (see Fig. S3).

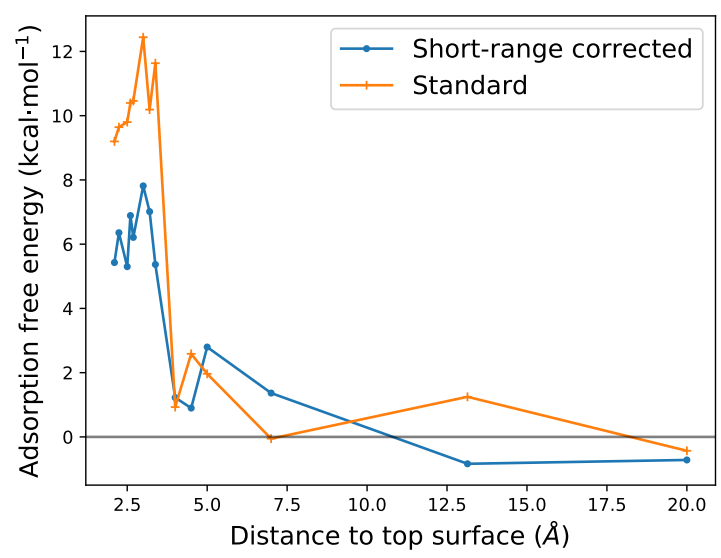

Figure 3: Adsorption free energy $\Delta_{a} G^{\text {solv }}$ for immobilized DFT water molecule on a 4 layers slab of $\mathrm{Pt}(111)$ using a $\mathrm{p}(4 \times 4)$ cell as a function of the distance of the oxygen to the surface. Its interactions with the slab are described at DFT level. Corrected refers to free energies computed with the coulombic correction proposed, in opposition to the uncorrected energies. Free energies are given an estimated $\pm 1.0 \mathrm{kcal} \cdot \mathrm{mol}^{-1}$ accuracy (See SI S4 for details).

According to Fig 3, $\Delta_{a} G^{\text {solv }}$ is positive at short distances and diminishes from 11 or $7 \mathrm{kcal} \cdot \mathrm{mol}^{-1}$ for the "standard" and "short-range corrected" Coulomb interactions, to about zero at long distance (> $8 \AA$ ) while consistently displaying a slight second peak of 2 $\mathrm{kcal} \cdot \mathrm{mol}^{-1}$ at $\sim 5 \AA$. The decay to zero after $8 \AA$ means that from this distance on, there is 
no interaction between the molecule and the surface, and, furthermore, that even the water organization around the frozen molecule is the same as in the bulk. In contrast, at short distances the three differences mentioned above and discussed in the next paragraphs might contribute to make $\Delta_{a} G^{\text {solv }}$ positive.

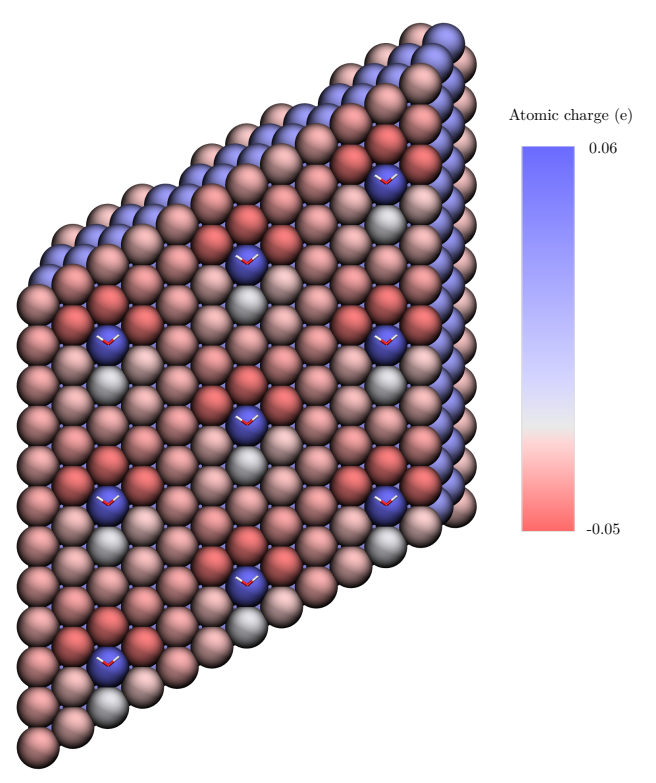

Figure 4: Atomic charges computed for the slab interacting with "DFT water" molecules. The charges are CM5 charges scaled by 1.27 according to ref 43 .

The obvious difference (i) is that the interaction of a MM molecule at the surface is replaced by $\Delta_{a} E_{D F T}^{v a c}$ for $\mathrm{H}_{2} \mathrm{O}^{\mathrm{DFT}} @ \mathrm{Pt}(111)$. However, this change is expected to be well described by the GAL17 forcefield, ${ }^{34}$ and should, therefore, not lead to a considerable contribution.

The subtle difference (ii) is connected to the atomic charges: the charges of $\mathrm{H}_{2} \mathrm{O}^{\mathrm{DFT}}$ and the metallic atoms are modified by polarization and non-negligible charge transfer at the DFT level, ${ }^{66}$ an effect that is only implicitly (at the single molecule level) taken into account in GAL17. Fig. 4 illustrates the DFT charge distribution at the DFT adsorption minimum. On average, the surface atoms are negatively charged, even though a strong positive charge is found for the Pt atom on which the oxygen is adsorbed. This positive charge is not very problematic in the MM computations since it is "shielded" by $\mathrm{H}_{2} \mathrm{O}^{\text {DFT }}$ and 
only MM water molecules in the second layer will be affected by it. The negative charge, which accumulates in the vicinity of the hydrogen atoms, can, however, lead to substantial interactions with the oxygen atoms of co-adsorbed MM water molecules. Even a partial charge of only $-0.05 e^{-}$at the adsorption minimum distance of of $2.5 \AA$ yields an artificial repulsion of about $5 \mathrm{kcal} \cdot \mathrm{mol}^{-1}$ with the water oxygen atom. It is this repulsion that the Coulomb short-range correction of Eq. 4 is designed to attenuate. Indeed, switching the correction on reduces $\Delta_{a} G^{\text {solv }}$ at short distance from $11 \mathrm{kcal} \cdot \mathrm{mol}^{-1}$ to $7 \mathrm{kcal} \cdot \mathrm{mol}^{-1}$. We, therefore, assign this $4 \mathrm{kcal} \cdot \mathrm{mol}^{-1}$ difference as the cost of the unequal description of charge transfer. While the DFT polarization is, of course, physically meaningful, the issue here is that the MM water molecules do neither lead to such a polarization nor is the force field (GAL17) adapted to deal with the surface polarization, but built for a neutral surface, as discussed in section 2.2.

Finally, point (iii) questions if keeping a water molecule frozen at the interface significantly disturbs the interfacial organization. To quantify this effect, we perform TIs with frozen MM molecules instead of $\mathrm{H}_{2} \mathrm{O}^{\text {DFT }}$. These tests show that a MM frozen water molecule results in a $\Delta_{a} G^{\text {solv }}$ very close to 0 , i.e., that freezing in itself does not introduce a significant artifact.

Having addressed the three points above, we may ask ourselves where the remaining 7 $\mathrm{kcal} \cdot \mathrm{mol}^{-1}$ at short distance come from. In the spirit of our tests with frozen MM water molecules, we perform further tests to assess the impact of the internal polarization of the water molecule upon adsorption. We observe that the oxygen atom in $\mathrm{H}_{2} \mathrm{O}^{\mathrm{DFT}} @ \mathrm{Pt}(111)$ is less negatively charged compared to the non-adsorbed water molecule (-0.7 vs. $\left.-0.8 e^{-}\right)$. Due to this weaker internal polarization, the interaction of $\mathrm{H}_{2} \mathrm{O}^{\text {DFT }}$ with the other water molecules at the interface is much weaker than it "should" be, resulting in the positive $\Delta_{a} G^{\text {solv }}$.

As a result of this analysis, we conclude that the Coulomb correction is an important component of solvation energies at metallic surfaces. However, direct solvent effects, i.e., 
the modification of water-adsorbate interaction due to the surface are only approximately taken into account, as shown here by the remaining free energy difference, and already highlighted during the development of GAL17. ${ }^{34}$ Thus, if suspected to be important, the direct water effects should be treated via microsolvation. ${ }^{15}$ Our hypothesis is, however, that the water-water interaction at the interface is among the worst cases possible, which means that we expect errors below $7 \mathrm{kcal} \cdot \mathrm{mol}^{-1}$ for general adsorbates.

\subsection{Solvation free energy of the $\operatorname{Pt}(111)$ surface}

In order to validate the scheme beyond the "intrinsic" worst-case scenario of section 4.1, we estimate the solvation free energy of the $\mathrm{Pt}(111)$ surface. This surface solvation free energy $\Gamma_{\text {surf }}$ (see Eq. S3 in the SI) corresponds to the free energy change associated with the creation of a water interface with a platinum slab in the middle of bulk water. In other words, the Pt-vacuum surface free energy does not contribute. It is, therefore, negative if the stabilizing interactions between water and platinum are strong enough to overcome the cost of creating a "gap" in water that will be filled with platinum. $\Gamma_{\text {surf }}$ can be computed similarly to the MMsolv term of Eq. 1 via SolvHybrid by adapting the latter to allow the creation of the full platinum slab instead of only the adsorbed molecule, with all the details exposed in the supplementary information (section S5). Using the TIP3P water model $\Gamma_{\text {surf }}$ is found to be $-0.46 \mathrm{~J} \cdot \mathrm{m}^{-2}\left(-5.2 \mathrm{kcal} \cdot \mathrm{mol}^{-1} \cdot\right.$ atom $\left.^{-1}\right)$. As a comparison, we also compute the corresponding value for the TIP4P water model and obtain $-0.51 \mathrm{~J} \cdot \mathrm{m}^{-2}$ $\left(-5.8 \mathrm{kcal} \cdot \mathrm{mol}^{-1} \cdot\right.$ atom $\left.^{-1}\right)$. The corresponding value for the water/gas-phase interface has been determined to $0.052 \mathrm{~J} \cdot \mathrm{m}^{-2},{ }^{67}$ i.e., about ten times less in absolute value and, of course, positive, as the creation of this interface costs energy. Compared to the value of $-0.32 \mathrm{~J} \cdot \mathrm{m}^{-2}$ reported by Campbell et. al. ${ }^{68}$ the accuracy of the result is very encouraging and on the same order of magnitude as the estimate of $0.27 \mathrm{~J} \cdot \mathrm{m}^{-2}$ by Gim et al. ${ }^{69}$ using a more sophisticated electrostatic embedding ${ }^{70}$ but a less accurate water-metal force field compared to this present study. The sign of this value is consistent with the wetability 
of platinum. The magnitude is almost reduced by a factor of two with respect to the adsorption energy of a single water molecule on a platinum slab (roughly $-9 \mathrm{kcal} \cdot \mathrm{mol}^{-1}$ according to DFT), which is coherent with the energetic and entropic cost to create the interface. The contribution due to the loss of water-water interactions due to the creation of the interface is, as stated above, only responsible for a reduction of about $0.5 \mathrm{kcal} \cdot \mathrm{mol}^{-1}$ with respect to the vacuum adsorption energy, highlighting the significant difference between the solid/liquid and liquid/gas interface.

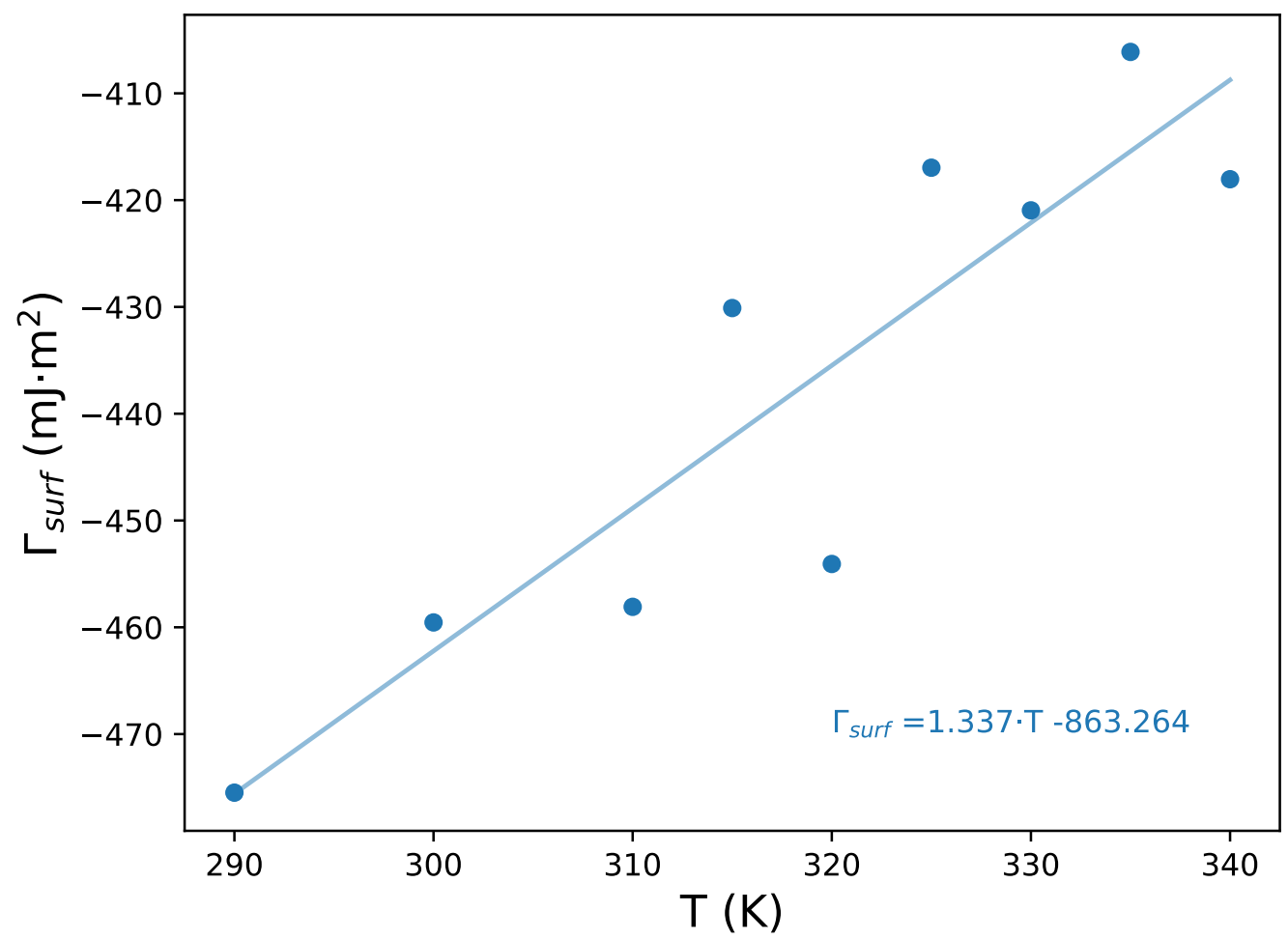

Figure 5: Solvation free energy of the $\operatorname{Pt}(111)$ surface in function of the temperature

A remarkable feature of explicit solvent molecular dynamics is the possibility to evaluate the solvation free energy as a function of the temperature. This contrasts with contiuum models such as PCM that are fitted for a given temperatures and cannot predict the temperature dependence. ${ }^{21}$ In practice, we evaluate $\Gamma_{\text {surf }}$ for a range of temperatures and determine the enthalpy and entropy according to its derivative (see Fig. 5). The enthalpic 
component $\Delta_{h} H_{\text {surf }}$ of the surface solvation free energy is closely related to the stabilizing interaction between Pt and water, while its entropic counterpart $-T \Delta_{h} S_{\text {surf }}$ is linked to the interfacial reorganization. Since the molecules are partially immobilized at the interface, a fast reorganizing (hot) solvent is expected to loose more entropy than a cold solvent upon the formation of the interface. Despite the statistical noise, $\Gamma_{\text {surf }}$ increases (becomes less negative) when increasing the temperature. The curve is fitted to a linear formula following $\Gamma_{\text {surf }}=\Delta_{h} H_{\text {surf }}-T \Delta_{h} S_{\text {surf }}$ with $\Delta_{h} H_{\text {surf }}=-0.86 \mathrm{~J} \cdot \mathrm{m}^{-2}$ and $\Delta_{h} S_{\text {surf }}=-1.34$ $\mathrm{mJ} \cdot \mathrm{m}^{-2} \cdot \mathrm{K}^{-1}\left(\mathrm{R}^{2}=0.81\right)$. The negative entropy confirms the reduced mobility of water at the interface.

In conclusion, the solvation free energy of $\mathrm{Pt}(111)$ obtained with GAL17 and the adopted strategy to carefully converge the thermodynamic integrations results in good agreement with experimental estimates. Furthermore, we provide new insights on the decomposition of the entropy/enthalpy balance at the solid/liquid interface. This makes us confident to tackle the next step which is the solvation free energy of adsorbates at the $\mathrm{Pt}(111) /$ water interface.

\subsection{Adsorption of benzene and phenol at the $\mathrm{Pt}(111) /$ water interface}

The adsorption free energy of benzene and phenol are studied to quantitatively evaluate the accuracy level of our QM/MM hybrid scheme compared to the experimental estimates from Campbell et. al. ${ }^{68}$ This provides us also the opportunity to compare MMSolv solvation energies to the one obtained with the widely used PCM, implemented in VASPsol. ${ }^{21}$

As a start, we compare the solvation energies of molecules in solution to their experimental counterparts, taken from the Minnesota database of solvation free energies. ${ }^{71}$ The experimental standard solvation free energies of water, phenol and benzene in water are reported to be $-6.31,-6.62$ and $-0.87 \mathrm{kcal} \cdot \mathrm{mol}^{-1}$, respectively. The implicit solvent, which is fitted on this kind of reference data, is in qualitative agreement, yielding -7.5, -5.7 and $-1.0 \mathrm{kcal} \cdot \mathrm{mol}^{-1}$. The MMsolv bulk solvation energies $\Delta_{h} G_{M M}(M)$ are $-4.8,-4.9$ and 
$-2.3 \mathrm{kcal} \cdot \mathrm{mol}^{-1}$. While these solvation free energies are not highly accurate, although in agreement with previous reports, ${ }^{27,29}$ the error remains in the $1-2 \mathrm{kcal} \cdot \mathrm{mol}^{-1}$ range, which is acceptable in our context and comparable to PCM values. Such errors have also been proposed to originate from missing polarization and charge-transfer effects at the MM level. ${ }^{29}$ These computations also demonstrate that the computational setup is reasonable, i.e., (a) a sound combination of atomic charges and Lennard-Jones parameters and (b) that the direct space computation of the Coulomb interaction does not introduce major inaccuracies or inconsistencies.

Since MM naturally includes the size of the solvent molecules, we can expect that the coverage-dependence of adsorption energies differs between PCM and MMSolv. The aromatic molecules have been suggested to adsorb flat on the so-called bridge position at the $\mathrm{Pt}(111) /$ water interface. ${ }^{68,72}$ To investigate the coverage dependence and the preferred adsorption mode, we consider four different coverages (from 1/7 to 1/36 monolayer (ML), where the ML is defined with respect to the number of surface Pt atoms) for the two most stable gas-phase adsorption modes, as proposed by Chaudhary et.al. (see Fig. S4 in the SI) ${ }^{73}$ Since the relative stability of these two modes is conserved after the evaluation of solvation free energy, only the most stable mode are discussed below. We note that defining the coverage in terms of surface Pt atoms is most convenient as the saturation coverage is not unambiguously known. However, using this definition, care should be applied when comparing the coverage dependent adsorption energies of various molecules, as adding a small additional molecule (e.g. CO) has not the same effect as adding a large one (e.g. naphtalene). As described below, the size of the adsorbate also displaces a varying amount of water molecules from the first interfacial layer.

The adsorption free energies of phenol and benzene as a function of the coverage are reported in Fig 6, where we compare gas-phase DFT to PCM and our QM/MM hybrid level. For all cases considered, a difference of less than $10 \mathrm{kcal} \cdot \mathrm{mol}^{-1}$ is observed between the adsorption free energy computed in the gas-phase and the implicit solvent. Qualitatively, 


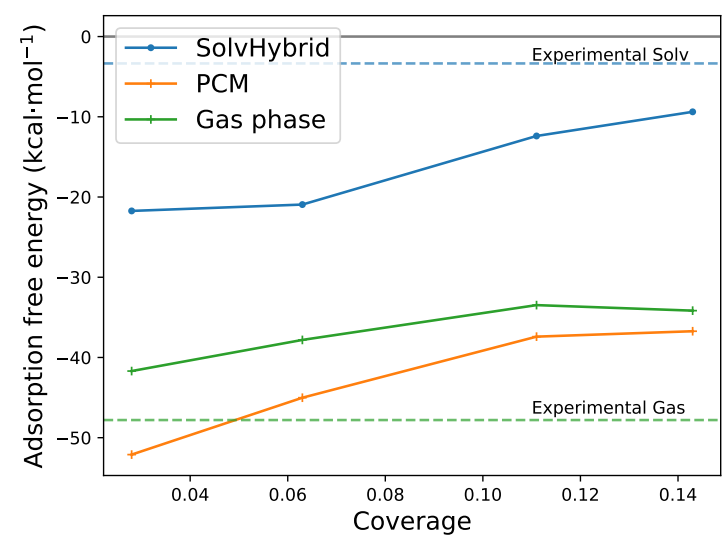

(a) Benzene

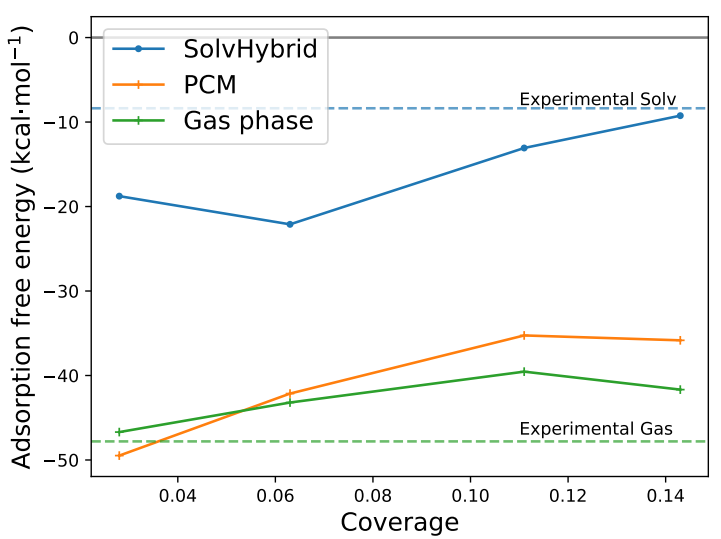

(b) Phenol

Figure 6: Adsorption free energy $\Delta_{a} G^{\text {solv }}$, calculated with SolvHybrid or using the Polarizable Continuum Model (PCM) as an implicit solvent, for a benzene or phenol molecule at 4 different coverages on a (111) platinum slab. Gas phase result refers to DFT-based adsorption energy for the same systems. The molecule were all adsorbed on position $\mathrm{Brg} 30^{\circ}$ for Benzene and Brg30 ${ }^{\circ} \mathrm{C} 2$ for Phenol (see Fig. S4). The dashed lines correspond to the experimental values of ref, ${ }^{71}$ whose precise coverage is unknown, with gas referring to gas phase adsorption energy and Solv to the free energy of adsorption in water.

PCM even stabilizes the adsorption of benzene at the $\operatorname{Pt}(111)$ interface, presumably due to the non-zero surface dipole moment obtained with the deformed benzene molecule (see Fig. 7) In contrast, the hybrid QM/MM adsorption free energy is reduced (in absolute value) by about $20 \mathrm{kcal} \cdot \mathrm{mol}^{-1}$ with respect to the gas-phase.

Campbell and co-workers have estimated the standard adsorption free energy (corresponding to $\sim 0.05 \mathrm{ML}$ ) of benzene and phenol at the $\mathrm{Pt}(111) /$ water interface to be $-3.4 \mathrm{kcal} \cdot \mathrm{mol}^{-1}$ and $-8.4 \mathrm{kcal} \cdot \mathrm{mol}^{-1}$, respectively. This compares to their adsorption heat, measured both at about $-47.8 \mathrm{kcal} \cdot \mathrm{mol}^{-1}$ at the $\mathrm{Pt}(111)$ gas-phase interface. Therefore, the trend between the gas-phase and aqueous-phase interface is qualitatively reproduced by our MMSolv results. In contrast, PCM predicts either no change (phenol) or a qualitatively wrong trend (benzene) and can, therefore, not be recommended to capture solvation effects at the $\mathrm{Pt}(111) /$ water interface, as also pointed out by Skylaris and co-workers. ${ }^{74}$

In Fig. 6 we draw a dashed horizontal line for the experimentally estimated adsorption free energy. This does not indicate that the experimental value is assumed to be a constant 
as a function of the coverage. Indeed, for the gas-phase it is known that this is not the case. ${ }^{8}$ At the solid/liquid interface, however, the coverage dependence is not known and, furthermore, Campbell and co-workers have argued that the experimental values are most likely compatible with island formation, i.e., locally much higher/lower coverages than on average. It is, therefore, difficult to judge the quantitative agreement between the experimental low-coverage, island-forming estimate and the homogeneous coverages investigated theoretically. As discussed in the section S7 in the SI, we have attempted to quantify the island-formation propensity according to a very simplified model. However, since applying the same framework to the gas-phase data leads to the prediction of island-formation, which goes against experiment evidence, ${ }^{75}$ we conclude that the model is oversimplified. Hence, we conclude that the hybrid results of Fig 6 are just in qualitative agreement with experiment (especially at high coverage), but that a quantitative assessment of the coverage dependence is beyond the scope of this investigation.

The use of explicit solvent and extensive phase-space sampling also allows to quantify the coverage-dependent number of water molecules co-adsorbed with benzene or phenol. As an example Fig. 7 shows that most Pt top sites not directly covered by the adsorbate are occupied with water in the presence of $0.0625 \mathrm{ML}$ benzene.

An analysis for 15 evenly spaced snapshots (corresponding to 300 ps of MD) of the first water layer (up to $4.5 \AA$ above the topmost Pt atom) provides quantitative results as a function of the coverage. For benzene, a water coverage of $0.02,0.32,0.55$, and $0.81 \mathrm{ML}$ is observed for a benzene coverage of $0.14,0.11,0.06$, and $0.03 \mathrm{ML}$, respectively which represent a nice linear decrease following approximately $\theta_{\text {water }}=1-6.5 * \theta_{\text {Benzene }}=$ $\left(n_{\text {top }}-6.5 * n_{\text {Benzene }}\right) / n_{\text {top }}\left(\mathrm{R}^{2}=0.99\right)$. From this we deduce that each adsorbed benzene molecule displaces 6.5 water molecules at the interface. Similarly, water coverages of 0.01 , $0.17,0.53$, and $0.80 \mathrm{ML}$ were found for phenol, leading to a slope of $-6.9\left(\mathrm{R}^{2}=0.99\right)$. In agreement with chemical intuition, this demonstrates that phenol takes somewhat more space on the surface than benzene. The fact that the difference is only small is likely to 


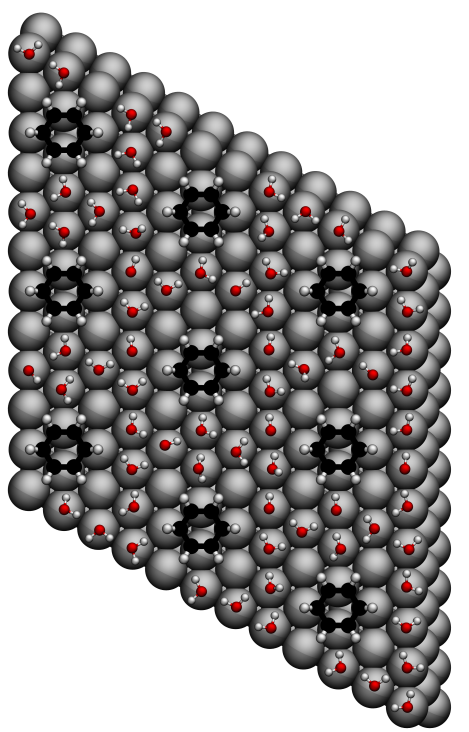

Figure 7: Snapshot of the first solvation layer extracted from the production run of the computation of $\Delta_{a} G^{\text {solv }}$ of the benzene.

be attributed to the possibility of the hydroxyl group of phenol to be incorporated into the hydrogen-bond network of the first water layer. Furthermore, this quantification of the water displacement, together with a tentative $e^{76,77}$ decomposition of the solvation free energy change into an electrostatic and non-electrostatic component (see Table S1 in the SI) provides a strong evidence that the qualitative difference between the implicit solvation models and our explicit solvation scheme are related to the non-electrostatic energy terms associated with the displacement of water molecules upon adsorption.

On the one hand, these results illustrate the accuracy of our hybrid QM/MM scheme to predict experimental adsorption energies at the $\mathrm{Pt}(111)$ / water interface with a far better accuracy than the commonly used PCM. On the other hand, the explicit description and extensive phase-space sampling provides physical insight, such as the entropy/enthalpy balance at the interface and the competition between water and other adsorbates. In summary, our scheme is very promising and seems mostly limited by the accuracy of the MM energy expression, which can, however, be further improved without the need of adjustments in the adopted strategy. Hence we foresee a bright future for the SolvHybrid package for future investigations of solvation effects at the solid/liquid interface. 


\section{Conclusion}

Alchemical transformations are powerful to determine solvation free energies at the molecular mechanics level of theory. As we have demonstrated herein, the near-chemisorption interaction of water with a prototypical $\mathrm{Pt}(111)$ surface necessitates adjustments of the "standard" thermodynamic cycle in order to reproducibly avoid hysteresis when computing the solvation free energies at the $\mathrm{Pt} /$ water interface. In our approach, this involves an intermediate state with weak interactions between the water solvent and the metal surface. Furthermore, the electrostatic interactions between the strongly adsorbed water molecules and the metal atoms is damped in order to avoid nonphysical contributions due to double counting. The resulting scheme, implemented in the freely available SolvHybrid software package that currently relies on the combination of VASP and AMBER, reliably determines solvation free energies at the $\mathrm{Pt} /$ water interface. It is validated by the assessment of the solvation free energy of the $\mathrm{Pt}(111)$ surface, which is, with $-0.46 \mathrm{~J} \cdot \mathrm{m}^{-2}$ for the TIP3P water model, in good agreement with the experimental estimate of $-0.32 \mathrm{~J} \cdot \mathrm{m}^{-2}$. More importantly, combined with DFT adsorption energies in gas-phase, adsorption energies from the bulk solution to the $\mathrm{Pt} /$ water interface are obtained at a hybrid DFT-MM level. As an example, a reduction of $\sim 30 \mathrm{kcal} \cdot \mathrm{mol}^{-1}$ is obtained for the adsorption of benzene and phenol at the $\operatorname{Pt}(111) /$ water interface compared to the gas-phase. This compares well with the experimental estimates of $\sim 40 \mathrm{kcal} \cdot \mathrm{mol}^{-1}$, while the implicit solvent estimate of $\sim 0$ is qualitatively incorrect. The major contribution at the hybrid level is traced back to the competition of the adsorbate with water molecules: the adsorption of a single aromatic molecule expels six to seven water molecules from the strongly bound first water layer. A further advantage of our approach compared to implicit solvents is that the explicit phase-space sampling with an atomistic water model naturally includes coverage and temperature effects, so that they can be conveniently quantified. In summary, our QMMM hybrid scheme provides a routinely available semi-quantitative determination of adsorption energies at the metal/water interface, valuable in various contexts ranging 
from corrosion to liquid-phase heterogeneous catalysis.

\section{Acknowledgement}

This work benefited from the support of the project MuSiC ANR-14-CE06-0030 of the French National Research Agency (ANR). The authors thank the SYSPROD project and AXELERA Pôle de Compétitivité for financial support (PSMN Data Center). AWG would like to acknowledge support by the National Science Foundation (grant CHE-1416571).

\section{Supporting Information Available}

The supplementary material contains additional Figures and Tables, as well as the DFT coordinates of all systems considered.

\section{References}

(1) Schiros, T.; Andersson, K.; Pettersson, L.; Nilsson, A.; Ogasawara, H. Chemical bonding of water to metal surfaces studied with core-level spectroscopies. Journal of Electron Spectroscopy and Related Phenomena 2010, 177, 85-98.

(2) Brown, W. A.; Kose, R.; King, D. A. Femtomole Adsorption Calorimetry on SingleCrystal Surfaces. Chemical Reviews 1998, 98, 797-832.

(3) Besson, M.; Gallezot, P.; Pinel, C. Conversion of Biomass into Chemicals over Metal Catalysts. Chemical Reviews 2014, 114, 1827-1870.

(4) Liu, L.; Corma, A. Metal Catalysts for Heterogeneous Catalysis: From Single Atoms to Nanoclusters and Nanoparticles. Chemical Reviews 2018, 118, 4981-5079. 
(5) Seh, Z. W.; Kibsgaard, J.; Dickens, C. F.; Chorkendorff, I.; Nørskov, J. K.; Jaramillo, T. F. Combining theory and experiment in electrocatalysis: Insights into materials design. Science 2017, 355, eaad4998.

(6) Chibani, S.; Michel, C.; Delbecq, F.; Pinel, C.; Besson, M. On the key role of hydroxyl groups in platinum-catalysed alcohol oxidation in aqueous medium. Catalysis Science E Technology 2013, 3, 339-350.

(7) Gu, Q.; Sautet, P.; Michel*, C. Unraveling the Role of Base and Catalyst Polarization in Alcohol Oxidation on Au and Pt in Water. ACS Catalysis 2018, 8, 11716-11721, Times Cited: 7.

(8) Carey, S. J.; Zhao, W.; Mao, Z.; Campbell, C. T. Energetics of Adsorbed Phenol on $\mathrm{Ni}(111)$ and $\operatorname{Pt}(111)$ by Calorimetry. The Journal of Physical Chemistry C 2019, 123, $7627-7632$.

(9) Bockris, J.; Jeng, K. In-situ studies of adsorption of organic compounds on platinum electrodes. Journal of Electroanalytical Chemistry 1992, 330, 541-581.

(10) Lu, F.; Salaita, G. N.; Laguren-Davidson, L.; Stern, D. A.; Wellner, E.; Frank, D. G.; Batina, N.; Zapien, D. C.; Walton, N.; Hubbard, A. T. Characterization of hydroquinone and related compounds adsorbed at $\mathrm{Pt}(111)$ from aqueous solutions: electron energy-loss spectroscopy, Auger spectroscopy, LEED, and cyclic voltammetry. Langmuir 1988, 4, 637-646.

(11) Michel, C.; Zaffran, J.; Ruppert, A. M.; Matras-Michalska, J.; Jedrzejczyk, M.; Grams, J.; Sautet, P. Role of water on metal catalyst performance for ketone hydrogenation. A join experimental and theoretical study on levulinic acid conversion into gammavalerolactone. Chem. Comm. 2014, 50, 12450.

(12) Michel, C.; Auneau, F.; Delbecq, F.; Sautet, P. C-H versus O-H Bond Dissociation 
for Alcohols on a $\mathrm{Rh}(111)$ Surface: A Strong Assistance from Hydrogen Bonded Neighbors. ACS Catal. 2011, 1, 1430-1440.

(13) Hibbitts, D. D.; Loveless, B. T.; Neurock, M.; Iglesia, E. Mechanistic Role of Water on the Rate and Selectivity of Fischer-Tropsch Synthesis on Ruthenium Catalysts. Angew. Chem. Int. Ed. 2013, 52, 12273-12278.

(14) Garcia-Rates, M.; Garcia-Muelas, R.; Lopez, N. Solvation Effects on Methanol Decomposition on $\operatorname{Pd}(111), \operatorname{Pt}(111)$, and $\mathrm{Ru}(0001)$. J. Phys. Chem. C 2017, 121, 13803-13809.

(15) Schweitzer, B.; Steinmann, S. N.; Michel, C. Can microsolvation effects be estimated from vacuum computations? A case-study of alcohol decomposition at the H2O/Pt(111) interface. Phys. Chem. Chem. Phys. 2019, 21, 5368-5377.

(16) Gao, W.; Keith, J. A.; Anton, J.; Jacob, T. Theoretical Elucidation of the Competitive Electro-oxidation Mechanisms of Formic Acid on Pt(111). Journal of the American Chemical Society 2010, 132, 18377-18385.

(17) Behtash, S.; Lu, J.; Mamun, O.; Williams, C. T.; Monnier, J. R.; Heyden, A. Solvation Effects in the Hydrodeoxygenation of Propanoic Acid over a Model Pd(211) Catalyst. J. Phys. Chem. C 2016, 120, 2724-2736.

(18) Gu, G. H.; Schweitzer, B.; Michel, C.; Steinmann, S. N.; Sautet, P.; Vlachos, D. G. Group Additivity for Aqueous Phase Thermochemical Properties of Alcohols on $\mathrm{Pt}(111)$. J Phys Chem C 2017, 10.1021/acs.jpcc.7b07340, Publisher: American Chemical Society.

(19) Faheem, M.; Saleheen, M.; Lu, J.; Heyden, A. Ethylene glycol reforming on Pt(111): first-principles microkinetic modeling in vapor and aqueous phases. Catal. Sci. Technol. 2016, 6, 8242-8256, Publisher: The Royal Society of Chemistry.

(20) Steinmann, S. N.; Sautet, P. Assessing a First-Principles Model of an Electrochemical 
Interface by Comparison with Experiment. J. Phys. Chem. C 2016, 120, 5619-5623, Publisher: American Chemical Society.

(21) Mathew, K.; Sundararaman, R.; Letchworth-Weaver, K.; Arias, T. A.; Hennig, R. G. Implicit solvation model for density-functional study of nanocrystal surfaces and reaction pathways. The Journal of Chemical Physics 2014, 140, 084106.

(22) Desai, S. K.; Pallassana, V.; Neurock, M. A Periodic Density Functional Theory Analysis of the Effect of Water Molecules on Deprotonation of Acetic Acid over Pd(111). J. Phys. Chem. B 2001, 105, 9171-9182, Publisher: American Chemical Society.

(23) Yoon, Y.; Rousseau, R.; Weber, R. S.; Mei, D.; Lercher, J. A. First-Principles Study of Phenol Hydrogenation on Pt and Ni Catalysts in Aqueous Phase. Journal of the American Chemical Society 2014, 136, 10287-10298.

(24) Bellarosa, L.; García-Muelas, R.; Revilla-López, G.; López, N. Diversity at the Water-Metal Interface: Metal, Water Thickness, and Confinement Effects. ACS Central Science 2016, 2, 109-116.

(25) Faheem, M.; Heyden, A. Hybrid Quantum Mechanics/Molecular Mechanics Solvation Scheme for Computing Free Energies of Reactions at Metal-Water Interfaces. J. Chem. Theo. Comput. 2014, 10, 3354-3368, Publisher: American Chemical Society.

(26) Saleheen, M.; Zare, M.; Faheem, M.; Heyden, A. Computational Investigation of Aqueous Phase Effects on the Dehydrogenation and Dehydroxylation of Polyols over Pt(111). J. Phys. Chem. C 2019, 123, 19052-19065.

(27) Steinmann, S. N.; Sautet, P.; Michel, C. Solvation free energies for periodic surfaces: comparison of implicit and explicit solvation models. Physical Chemistry Chemical Physics 2016, 18, 31850-31861. 
(28) Bodenschatz, C. J.; Sarupria, S.; Getman, R. B. Molecular-Level Details about Liquid H ${ }_{2}$ O Interactions with CO and Sugar Alcohol Adsorbates on Pt(111) Calculated Using Density Functional Theory and Molecular Dynamics. The Journal of Physical Chemistry C 2015, 119, 13642-13651.

(29) Zhang, X.; DeFever, R. S.; Sarupria, S.; Getman, R. B. Free Energies of Catalytic Species Adsorbed to Pt(111) Surfaces under Liquid Solvent Calculated Using Classical and Quantum Approaches. Journal of Chemical Information and Modeling 2019, 59, 21902198.

(30) Spohr, E.; Heinzinger, K. Molecular dynamics simulation of a water/metal interface. Chem. Phys. Letters 1986, 123, 218-221.

(31) Siepmann, J. I.; Sprik, M. Influence of surface topology and electrostatic potential on water/electrode systems. The Journal of Chemical Physics 1995, 102, 511-524.

(32) Limmer, D. T.; Willard, A. P.; Madden, P.; Chandler, D. Hydration of metal surfaces can be dynamically heterogeneous and hydrophobic. Proceedings of the National Academy of Sciences 2013, 110, 4200-4205.

(33) Heinz, H.; Vaia, R. A.; Farmer, B. L.; Naik, R. R. Accurate Simulation of Surfaces and Interfaces of Face-Centered Cubic Metals Using 12-6 and 9-6 Lennard-Jones Potentials. The Journal of Physical Chemistry C 2008, 112, 17281-17290.

(34) Steinmann, S. N.; Ferreira De Morais, R.; Götz, A. W.; Fleurat-Lessard, P.; Iannuzzi, M.; Sautet, P.; Michel, C. Force Field for Water over Pt(111): Development, Assessment, and Comparison. Journal of Chemical Theory and Computation 2018, 14, 3238-3251.

(35) Clabaut, P.; Fleurat-Lessard, P.; Michel, C.; Steinmann, S. N. Ten Facets, One Force Field: The GAL19 Force Field for Water-Noble Metal Interfaces. J. Chem. Theory Comput. 2020, Publisher: American Chemical Society. 
(36) Réocreux, R.; Michel, C.; Fleurat-Lessard, P.; Sautet, P.; Steinmann, S. N. Evaluating Thermal Corrections for Adsorption Processes at the Metal/Gas Interface. J. Phys. Chem. C 2019, 123, 28828-28835.

(37) Kresse, G. Ab initio molecular dynamics for liquid metals. Journal of Non-Crystalline Solids 1995, 192-193, 222-229.

(38) Kresse, G.; Furthmüller, J. Efficient iterative schemes for ab initio total-energy calculations using a plane-wave basis set. Physical Review B 1996, 54, 11169-11186.

(39) Case, D. A.; Cheatham, T. E.; Darden, T.; Gohlke, H.; Luo, R.; Merz, K. M.; Onufriev, A.; Simmerling, C.; Wang, B.; Woods, R. J. The Amber biomolecular simulation programs. Journal of Computational Chemistry 2005, 26, 1668-1688.

(40) D.A. Case, R.M. Betz, W. Botello-Smith, D.S. Cerutti, T.E. Cheatham, III, T.A. Darden, R.E. Duke, T.J. Giese, H. Gohlke, A.W. Goetz, N. Homeyer, S. Izadi, P. Janowski, J. Kaus, A. Kovalenko, T.S. Lee, S. LeGrand, P. Li, C. Lin, T. Luchko, R. Luo, B. Madej, D. Mermelstein, K.M. Merz, G. Monard, H. Nguyen, H.T. Nguyen, I. Omelyan, A. Onufriev, D.R. Roe, A. Roitberg, C. Sagui, C.L. Simmerling, J. Swails, R.C. Walker, J. Wang, R.M. Wolf, X. Wu, L. Xiao, D.M. York and P.A. Kollman (2017), AMBER 2017, University of California, San Francisco.

(41) Rappe, A. K.; Casewit, C. J.; Colwell, K. S.; Goddard, W. A.; Skiff, W. M. UFF, a full periodic table force field for molecular mechanics and molecular dynamics simulations. Journal of the American Chemical Society 1992, 114, 10024-10035.

(42) Hirshfeld, F. L. Bonded-atom fragments for describing molecular charge densities. Theoretica Chimica Acta 1977, 44, 129-138.

(43) Vilseck, J. Z.; Tirado-Rives, J.; Jorgensen, W. L. Evaluation of CM5 Charges for Condensed-Phase Modeling. Journal of Chemical Theory and Computation 2014, 10, 2802-2812. 
(44) Mackerell, A. D. Empirical force fields for biological macromolecules: Overview and issues. J. Comput. Chem. 2004, 25, 1584-1604.

(45) Brooks, I., Charles L; Pettitt, B. M.; Karplus, M. Structural and energetic effects of truncating long ranged interactions in ionic and polar fluids. J. Chem. Phys. 1985, 5897.

(46) Steinbach, P. J.; Brooks, B. R. New spherical-cutoff methods for long-range forces in macromolecular simulation. J. Comput. Chem. 1994, 15, 667-683.

(47) Steinmann, S. N.; Fleurat-Lessard, P.; Götz, A. W.; Michel, C.; Ferreira de Morais, R.; Sautet, P. Molecular mechanics models for the image charge, a comment on "including image charge effects in the molecular dynamics simulations of molecules on metal surfaces". J. Comput. Chem. 2017, 38, 2127-2129.

(48) Jorgensen, W. L.; Chandrasekhar, J.; Madura, J. D.; Impey, R. W.; Klein, M. L. Comparison of simple potential functions for simulating liquid water. The Journal of Chemical Physics 1983, 79, 926-935.

(49) Ryckaert, J.-P.; Ciccotti, G.; Berendsen, H. J. Numerical integration of the cartesian equations of motion of a system with constraints: molecular dynamics of n-alkanes. Journal of Computational Physics 1977, 23, 327-341.

(50) Ryckaert, J. Special geometrical constraints in the molecular dynamics of chain molecules. Molecular Physics 1985, 55, 549-556.

(51) Berendsen, H. J. C.; Postma, J. P. M.; van Gunsteren, W. F.; DiNola, A.; Haak, J. R. Molecular dynamics with coupling to an external bath. The Journal of Chemical Physics 1984, 81, 3684-3690.

(52) Beutler, T. C.; Mark, A. E.; van Schaik, R. C.; Gerber, P. R.; van Gunsteren, W. F. Avoiding singularities and numerical instabilities in free energy calculations based on molecular simulations. Chemical Physics Letters 1994, 222, 529-539. 
(53) Zacharias, M.; Straatsma, T. P.; McCammon, J. A. Separation-shifted scaling, a new scaling method for Lennard-Jones interactions in thermodynamic integration. The Journal of Chemical Physics 1994, 100, 9025-9031.

(54) Perdew, J. P.; Wang, Y. Accurate and simple analytic representation of the electron-gas correlation energy. Physical Review B 1992, 45, 13244-13249.

(55) Perdew, J. P.; Burke, K.; Ernzerhof, M. Generalized Gradient Approximation Made Simple. Physical Review Letters 1996, 77, 3865-3868.

(56) Steinmann, S. N.; Corminboeuf, C. A generalized-gradient approximation exchange hole model for dispersion coefficients. The Journal of Chemical Physics 2011, 134, 044117.

(57) Gautier, S.; N. Steinmann, S.; Michel, C.; Fleurat-Lessard, P.; Sautet, P. Molecular adsorption at $\operatorname{Pt}(111)$. How accurate are DFT functionals? Physical Chemistry Chemical Physics 2015, 17, 28921-28930.

(58) Blochl, P. E. Projector augmented-wave method. 27.

(59) Kresse, G.; Joubert, D. From ultrasoft pseudopotentials to the projector augmentedwave method. Physical Review B 1999, 59, 1758-1775.

(60) Monkhorst, H. J.; Pack, J. D. Special points for Brillouin-zone integrations. Physical Review B 1976, 13, 5188-5192.

(61) Mathew, K.; Kolluru, V. S. C.; Mula, S.; Steinmann, S. N.; Hennig, R. G. Implicit self-consistent electrolyte model in plane-wave density-functional theory. J. Chem. Phys. 2019, 151, 234101.

(62) Neugebauer, J.; Scheffler, M. Adsorbate-substrate and adsorbate-adsorbate interactions of Na and K adlayers on Al(111). Physical Review B 1992, 46, 16067-16080. 
(63) Marenich, A. V.; Jerome, S. V.; Cramer, C. J.; Truhlar, D. G. Charge Model 5: An Extension of Hirshfeld Population Analysis for the Accurate Description of Molecular Interactions in Gaseous and Condensed Phases. Journal of Chemical Theory and Computation 2012, 8, 527-541.

(64) Schlick, T. Molecular Modeling and Simulation: An Interdisciplinary Guide: An Interdisciplinary Guide, 2nd ed.; Interdisciplinary Applied Mathematics; Springer-Verlag: New York, 2010.

(65) Steinbrecher, T.; Mobley, D. L.; Case, D. A. Nonlinear scaling schemes for LennardJones interactions in free energy calculations. The Journal of Chemical Physics 2007, 127, 214108.

(66) Staub, R.; Iannuzzi, M.; Khaliullin, R. Z.; Steinmann, S. N. Energy Decomposition Analysis for Metal Surface? @ SAdsorbate Interactions by Block Localized Wave Functions. Journal of Chemical Theory and Computation 2019, 15, 265-275.

(67) Vega, C.; de Miguel, E. Surface tension of the most popular models of water by using the test-area simulation method. J. Chem. Phys. 2007, 126, 154707, Publisher: American Institute of Physics.

(68) Singh, N.; Campbell, C. T. A Simple Bond-Additivity Model Explains Large Decreases in Heats of Adsorption in Solvents Versus Gas Phase: A Case Study with Phenol on $\mathrm{Pt}(111)$ in Water. ACS Catalysis 2019, 9, 8116-8127.

(69) Gim, S.; Cho, K. J.; Lim, H.-K.; Kim, H. Structure, Dynamics, and Wettability of Water at Metal Interfaces. Sci Rep 2019, 9, 14805.

(70) Fdez Galvan, I.; Sanchez, M.; Martin, M.; Olivares del Valle, F.; Aguilar, M. ASEP/MD: A program for the calculation of solvent effects combining QM/MM methods and the mean field approximation. Computer Physics Communications 2003, 155, 244-259. 
(71) Kelly, C. P.; Cramer, C. J.; Truhlar, D. G. SM6: A Density Functional Theory Continuum Solvation Model for Calculating Aqueous Solvation Free Energies of Neutrals, Ions, and Solute-Water Clusters. Journal of Chemical Theory and Computation 2005, 1, 11331152, Publisher: American Chemical Society.

(72) Singh, N.; Sanyal, U.; Fulton, J. L.; Gutiérrez, O. Y.; Lercher, J. A.; Campbell, C. T. Quantifying Adsorption of Organic Molecules on Platinum in Aqueous Phase by Hydrogen Site Blocking and in Situ X-ray Absorption Spectroscopy. ACS Catalysis 2019, 9, 6869-6881.

(73) Chaudhary, N.; Hensley, A.; Collinge, G.; Wang, Y.; McEwen, J.-S. CoverageDependent Adsorption of Phenol on $\mathrm{Pt}(111)$ from First Principles. The Journal of Physical Chemistry C 2020, 124, 356-362, Publisher: American Chemical Society.

(74) Bramley, G.; Nguyen, M.-T.; Glezakou, V.-A.; Rousseau, R.; Skylaris, C.-K. Reconciling Work Functions and Adsorption Enthalpies for Implicit Solvent Models: A Pt (111)/Water Interface Case Study. J. Chem. Theory Comput. 2020, 16, 2703-2715, Publisher: American Chemical Society.

(75) Ihm, H.; White, J. M. Stepwise Dissociation of Thermally Activated Phenol on Pt(111). The Journal of Physical Chemistry B 2000, 104, 6202-6211.

(76) Mark, A. E.; van Gunsteren, W. F. Decomposition of the Free Energy of a System in Terms of Specific Interactions: Implications for Theoretical and Experimental Studies. Journal of Molecular Biology 1994, 240, 167-176.

(77) Boresch, S.; Karplus, M. The Meaning of Component Analysis: Decomposition of the Free Energy in Terms of Specific Interactions. Journal of Molecular Biology 1995, 254, 801-807. 


\section{Graphical TOC Entry}

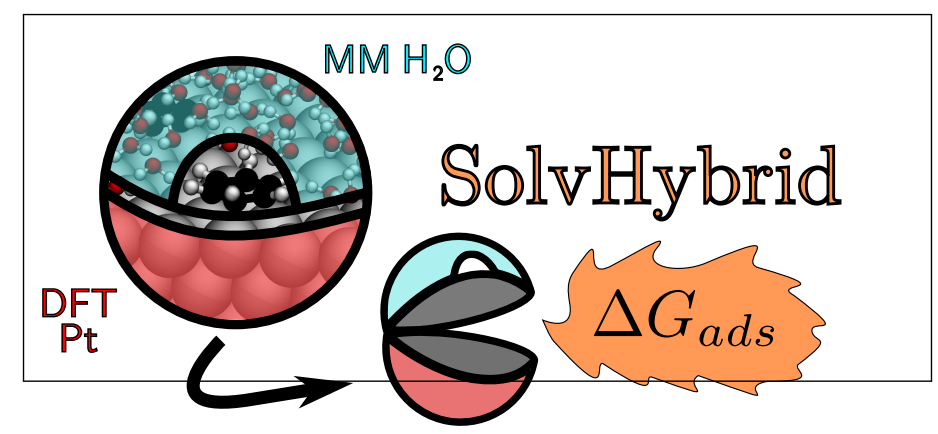

